\title{
Comparative Study between Clinical and Ultrasound Diagnosis of Emergency of Gynecology
}

\author{
HEND E. HASBY, M.Sc.*; ASHRAF E. GHARIEB, M.D.** and AHMED M. HAGRAS, M.D.** \\ The Departments of Emergency Medicine \& Traumatology* and Gynecology \& Obstetrics**, Faculty of Medicine, \\ Tanta University, Tanta, Egypt
}

\begin{abstract}
Background: Acute pelvic pain accounts for up to $40 \%$ of the visits to gynecological emergency departments and may indicate a serious condition. Potentially life-threatening gynecological emergencies are acute pelvic conditions that may spontaneously evolve into a life threatening situation. They may also carry a risk of sequelae (organ failure or organ removal) or death in the absence of prompt diagnosis and treatment.
\end{abstract}

The most common gynecological emergencies are ruptured ectopic pregnancy, adnexal torsion, and complicated pelvic inflammatory disease (tubo-ovarian abscess (TOA) and pyosalpinx). Missing these high-risk conditions may delay treatment that could lead to potentially negative patient outcomes.

Methods: This study was carried out over 1 year from September 2016 to September 2017 on 30 female patients. The records of all patients were review and data were collected prospectively. Our study included patients presented to the Emergency Department with gynecological emergencies.

These gynecological emergencies included patients presented with acute pelvic pain (e.g. ectopic pregnancy, torsion ovarian cyst, rupture ovarian cyst, pelvic inflammatory disease, tubo-ovarian abscess (TOA), and acute salpingitis) and/or vaginal bleeding (e.g. rupture uterus, and uterine tumors).

Results: Ectopic pregnancy was the most common gynecological emergency seen in our study (about $46.67 \%$ of patients). Other diagnosis found in this study were adnexal torsion (about $16.67 \%$ ), ovarian cyst rupture (about $13.33 \%$ ), PID (about 13.33\%), and uterine mass (about 10\%).

In this study, only about $53.33 \%$ of patients were diagnosed clinically before ultrasound was done. About $93.33 \%$ of patients were diagnosed after assessment by ultrasonography. There was statistically significant difference between clinical diagnosis and diagnosis after ultrasonography. $(p<0.05)$.

According to this study, there was a significant difference between clinical and radiological diagnosis using ultrasonography in diagnosis of ectopic pregnancy, adnexal torsion, and ovarian cyst rupture. $(p<0.05)$.

Correspondence to: Dr. Hend E. Hasby, E-Mail: hendhasby@yahoo.com
According to our data, physical examination cannot be used alone to safely rule out a surgical emergency in a woman presenting with acute pelvic pain. This suggests the benefit of adding bedside ultrasonography in the first-line diagnostic management of suspected gynecological emergencies.

Conclusion: Adding Ultrasonography as a bedside test was found to be superior to physical examination in diagnosis of acute pelvic pain. If a gynecologic disorder couldn't be confirmed, or the sonographic finding are equivocal, MultiDetector Computed Tomography (MDCT) is another imaging choice.

Ultrasound is generally accepted as the first imaging modality used in patients with acute pelvic pain. The true value of ultrasound in acute pelvic pain lies in its ability to detect gynecologic disorders.

Key Words: Ultrasound-Emergency-Gynecology.

\section{Introduction}

GYNECOLOGICAL emergencies are disease conditions of the female reproductive system that threaten the life of the woman, her sexual function and the perpetuation of her fertility [1].

Common gynecological emergencies present as acute pelvic/abdominal pain, abnormal vaginal bleeding, or both. The most common gynecological emergencies are ectopic pregnancy, acute pelvic inflammatory disease (PID), miscarriage, and ruptured or hemorrhagic ovarian cysts [2].

The differential diagnosis of abdominal/pelvic pain is broad and includes non-gynecological causes. These include gastrointestinal sources (e.g. appendicitis, Meckel's diverticulitis, Crohn's disease) and genitourinary conditions (e.g. distal urolithiasis, urinary tract infections) [3].

Acute pelvic pain is often associated with other nonspecific symptoms and signs such as nausea, 
vomiting. So, imaging is frequently required to narrow the differential diagnosis [3].

Diagnosis of gynecological emergencies is suspected on the basis of symptoms, physical evaluation (including abdominal, pelvic, and bimanual gynecological examinations), laboratory tests, and transvaginal ultrasonography (TVS) and/or transabdominal ultrasonography (TAS) [3]

Ultrasound (US) is the most widely accepted initial imaging modality of choice for female pelvis. It is widely available, familiar, and is relatively inexpensive. Higher resolution imaging of TVS provides high diagnostic accuracy for pelvic pathology $[4,5]$.

TVS should usually be used in conjunction with TAS. If examination by the ultrasound is nondiagnostic, computed tomography (CT) or magnetic resonance imaging (MRI) should be considered

If gynecologic cause of pelvic pain is confirmed, other imaging studies might be unnecessary, thereby reducing cost, and adverse complications of CT (contrast material reactions, and radiation exposure)

\section{Patients and Methods}

\section{Study design:}

A prospective randomized study was conducted in the Emergency Department (ED) of Tanta University Hospital. This study was carried out over 1 year from September 2016 to September 2017 on 30 female patients. The records of all patients were reviewed and data were collected prospectively. Our study included patients presented to the Emergency Department with gynecological emergencies. All patients underwent the standard procedures of the protocol.

\section{Ethics of the study:}

The Research Ethical Committee, Faculty of Medicine, Tanta University approved the design of the study. An informed written consent was obtained from all patients or from the relatives after full explanation of benefits and risks.

Privacy of all patient data was granted and there was code number for every patient file that includes all investigations.

\section{Study population:}

This study was carried out over 1 year from September 2016 to September 2017 on 30 female patients. The records of all patients were reviewed and data were collected prospectively. Our study included patients presented to the Emergency Department with gynecological emergencies.

These gynecological emergencies included patients presented with acute pelvic pain (e.g. ectopic pregnancy, torsion ovarian cyst, rupture ovarian cyst, pelvic inflammatory disease, tuboovarian abscess (TOA), and acute salpingitis) and/or vaginal bleeding (e.g. rupture uterus, and uterine tumors).

\section{Inclusion criteria:}

- Female patients with acute severe pelviabdominal pain.

- Female patients with acute severe vaginal bleeding.

\section{Exclusion criteria:}

- Impaired consciousness, disorientation.

- Inability to give informed consent.

- Healthy intrauterine pregnant females.

- Hemodynamically unstable patients.

All patients were subjected to the following:

A- Full history taking from the patient or her relatives: Including personal, menstrual, obstetric, medical and history of previous operations.

B- Analysis of the main complain of the patient: Acute Pelvic Pain: Included site, radiation, onset, course, duration, severity, associated symptoms. Vaginal Bleeding: Included color, onset, course, duration, amount.

C- Full clinical examination:

- General examination:

This included blood pressure, heart rate (HR), temperature, respiratory rate (RR), saturation of oxygen by pulse oximetry $\left(\mathrm{S}_{\mathrm{p}} \mathrm{O}_{2}\right)$.

- Abdominal examination:

- Inspection: This included abdominal scars, and ecchymosis.

- Palpation: This included tenderness, rebound tenderness, guard, and rigidity.

- Cardiovascular Examination.

- Chest Examination.

- Neurological Examination.

D- Investigations:

1- Routine laboratory investigation:

- Random blood sugar (RBS).

- Complete blood picture (CBC). 
- Kidney \& liver function tests.

- Serum electrolytes.

- Urine analysis.

2- Pelvi-abdominal ultrasound:

a- Transabdominal US (TAS): For the initial evaluation of the patient

b- Transvaginal US (TVS): When TAS was nonconclusive.

Technique of ultrasonography examinations of female pelvis:

Ultrasonography was done using Digital Ultrasonic Imaging System questionnaire with either satisfied or unsatisfied result.

\section{A- Transabdominal sonography (TAS): \\ Preparation:}

The urinary bladder should be distended sufficiently to see the uterus and adnexal areas. The full bladder displaces the bowel out of the pelvis. It also decreases uterine physiological anteversion, bringing it into a better position for ultrasound scanning. So, it becomes an acoustic window to view pelvic organs and pathology.
The bladder is considered optimally full when it covers the fundus of normal sized uterus. Overdistension is not recommended as it may compromise the sonographic evaluation and distort the anatomy of the pelvic organs.

Steps of TAS Examination: Are Any clothing, and other objects that may interfere with the scan were removed. The patient was placed in supine position on the examination table. A gel-like substance was then applied to the abdomen / pelvis.

The transducer (Transducer used in our study was C6-2) pressed against the skin and moved around over the area being studied. (Fig. 1).

The patients were examined in both longitudinal and transverse planes. Documentation of the right upper quadrant (Morison's pouch and subphrenic area) and bilateral renal areas were obtained (for the presence or absence of free fluid). Images of structures were displayed on the computer screen and was recorded. Once the procedure completed, the gel was removed.
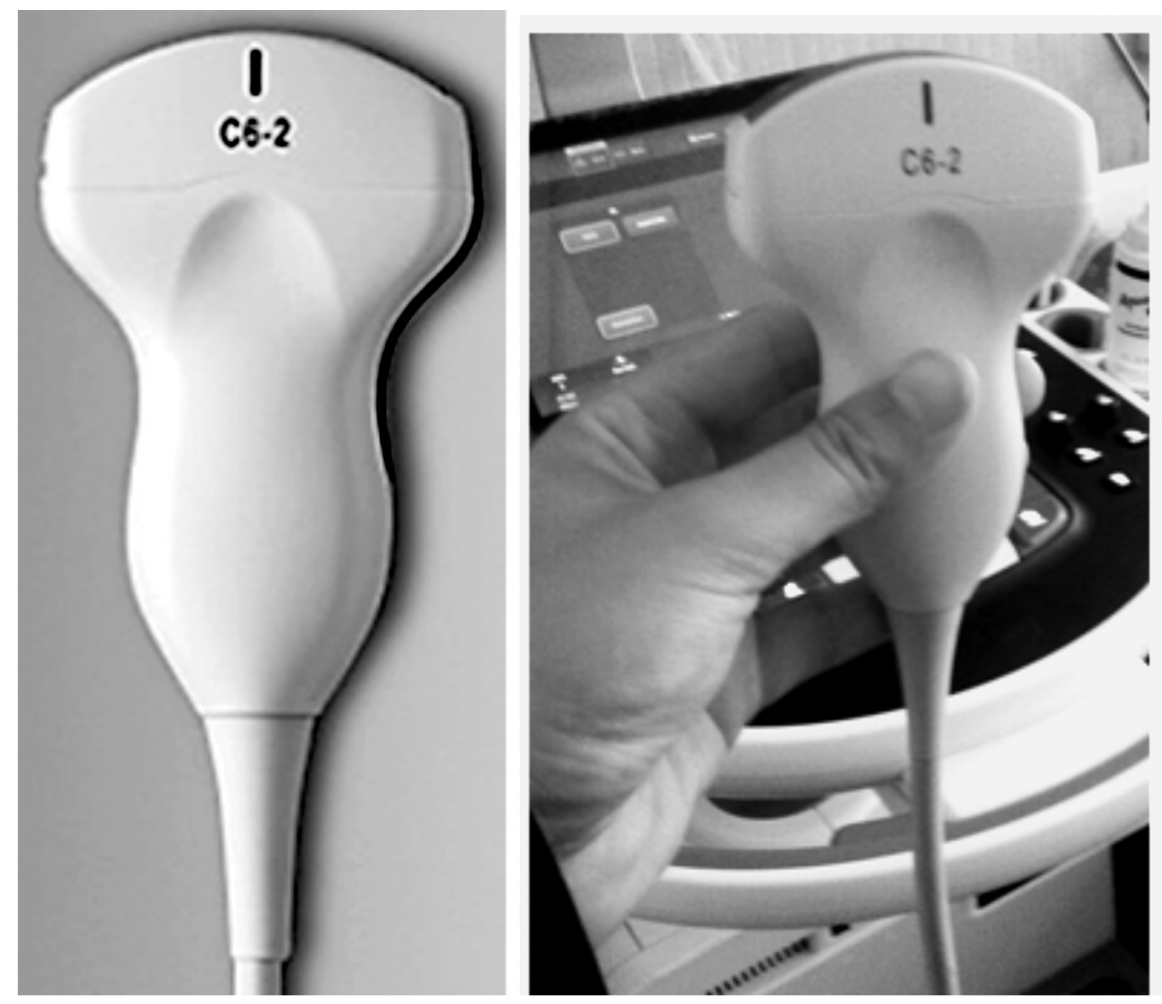

Fig. (1): Transducer Model C6-2. 
In some patients, the pelvic organs were not optimally visualized by transabdominal sonography secondary to:

- Uterine anomalies such as retroversion.

- Peritoneal adhesions.

- Overlying bowel gas.

In such cases, transvaginal sonography was performed and provided additional information when findings with transabdominal sonography were equivocal or confusing.

\section{B- Transvaginal sonography (TVS):}

\section{Preparations:}

After transabdominal study was completed, the patient was asked to empty the bladder to: Reduce the patient discomfort. And make the pelvic structures close to the transducer.

Steps of TVS Examination: The patient was asked to undress from the waist down and dressed a gown. The patient was placed in lithotomy position with her buttocks elevated. The elevation was necessary to provide adequate motility of the transducer handle A long, thin transvaginal transducer was covered with latex sheath and lubricated. The tip of the transducer was inserted into the vagina (may be introduced by the patient or the sonographer). The transducer was gently turned and angled to bring the areas for study into focus. Images of structures were displayed on the computer screen, and was recorded.

\section{Results}

Final diagnosis of patients was one of those diseases (ectopic pregnancy, adnexal torsion, ovarian cyst rupture, pelvic inflammatory disease "PID", or uterine mass).

Records and data of all patients were collected prospectively. The results were summarized, tabulated and statistically analyzed in the following tables and figures.

\section{Complaint:}

In our study, about $76.67 \%$ of patients presented mainly with acute pelvic pain. About $23.33 \%$ of patients presented mainly with vaginal bleeding.

Table (1): Complaint of the studied patients.

\begin{tabular}{lll}
\hline Complaint & n. & $\%$ \\
\hline Pain & 23 & 76.67 \\
Bleeding & 7 & 23.33 \\
\hline Total & 30 & 100 \\
\hline
\end{tabular}

In ectopic pregnancy, about $64.29 \%$ of patients presented with acute pelvic pain, and about $35.71 \%$ of patients presented with vaginal bleeding. In uterine mass, about $33.33 \%$ of patients presented with acute pelvic pain, and about $66.67 \%$ of patients presented with vaginal bleeding.

In patients with PID, ovarian cyst rupture and adnexal torsion, all patients were presented mainly with acute pelvic pain.

Table (2): Complaint of the patients in each diagnosis.

\begin{tabular}{llc}
\hline Complaint & \multicolumn{1}{c}{ Pain } & Bleeding \\
\hline Ectopic pregnancy [n. (\%)] & $9(64.29)$ & $5(35.71)$ \\
Adnexal Torsion [n. (\%)] & $5(100)$ & \\
Ovarian Cyst Rupture [n. (\%)] & $4(100)$ & - \\
PID [n. (\%)] & $4(100)$ & \\
Uterine [n. (\%)] & $1(33.33)$ & $2(66.67)$ \\
\hline Total [n. (\%)] & $23(76.67)$ & $7(23.33)$ \\
\hline
\end{tabular}

\section{Final diagnosis:}

In our study, the most common gynecological emergency was ectopic pregnancy. About $46.67 \%$ of patients were diagnosed as ectopic pregnancy, $16.67 \%$ of patients were diagnosed as adnexal torsion, $13.33 \%$ of patients were diagnosed as ovarian cyst rupture, $13.33 \%$ of patients were diagnosed as PID, and $10 \%$ of patients were diagnosed as uterine mass.

Table (3): Final diagnosis of the studied patients.

\begin{tabular}{lll}
\hline Final Diagnosis & $\mathrm{n}$. & $\%$ \\
\hline Ectopic Pregnancy & 14 & 46.67 \\
Adnexal Torsion & 5 & 16.67 \\
Ovarian Cyst Rupture & 4 & 13.33 \\
PID & 4 & 13.33 \\
Uterine Mass & 3 & 10 \\
\hline Total & 30 & 100 \\
\hline
\end{tabular}

Clinical diagnosis (before ultrasonography):

In our study, only about $53.33 \%$ of patients were diagnosed clinically before ultrasound was done.

Table (4): Clinical diagnosis of the studied patients.

\begin{tabular}{lcc}
\hline Clinical Diagnosis & N. & $\%$ \\
\hline Diagnosed & 16 & 53.33 \\
Not Diagnosed & 14 & 46.67 \\
\hline Total & 30 & 100 \\
\hline
\end{tabular}


In our study, about $62.5 \%$ of the diagnosed patients were ectopic pregnancy, $12.5 \%$ of the diagnosed patients were adnexal torsion, $6.25 \%$ of the diagnosed patients were ovarian cyst rupture, $12.5 \%$ of the diagnosed patients were PID, and $6.25 \%$ of the diagnosed patients were uterine mass.

\section{Radiological diagnosis (after ultrasonography):}

In our study, about $93.33 \%$ of patients were diagnosed after assessment by ultrasonography.

Table (5): Diagnosis after ultrasonography of the studied patients.

\begin{tabular}{lll}
\hline Ultrasonographic & n. & $\%$ \\
Diagnosis & 28 & 93.33 \\
Diagnosed & 2 & 6.67 \\
Not Diagnosed & & \\
\hline Total & 30 & 100 \\
\hline
\end{tabular}

In our study after ultrasonography, about $50 \%$ of the diagnosed patients were ectopic pregnancy, $17.86 \%$ of the diagnosed patients were adnexal torsion, $14.29 \%$ of the diagnosed patients were ovarian cyst rupture, $10.71 \%$ of the diagnosed patients were PID, and $7.14 \%$ of the diagnosed patients were uterine mass.

Table (6): Diagnosis after ultrasonography of patients in each final diagnosis.

\begin{tabular}{llllll}
\hline \multirow{2}{*}{$\begin{array}{l}\text { Diagnosis by } \\
\text { Ultrasonography }\end{array}$} & \multicolumn{2}{c}{ Diagnosed } & $\begin{array}{c}\text { Not } \\
\text { Diagnosed }\end{array}$ & Total \\
\cline { 2 - 6 } & $\mathrm{n}$. & $\%$ & $\mathrm{n}$. & $\%$ & $\mathrm{n}$. \\
\hline Ectopic pregnancy & 14 & 50 & - & - & 14 \\
Adnexal Torsion & 5 & 17.86 & & & 5 \\
Ovarian Cyst Rupture & 4 & 14.29 & & & 4 \\
PID & 3 & 10.71 & 1 & 50 & 4 \\
Uterine Mass & 2 & 7.14 & 1 & 50 & 3 \\
\hline Total & 28 & 100 & 2 & 100 & 30 \\
\hline
\end{tabular}

\section{Diagnosis before and after ultrasonography:}

In all the studied patients:

Before ultrasound, about $53.33 \%$ of patients were diagnosed by clinical assessment. After ultrasound, about $93.33 \%$ of patients were diagnosed. There was statistically significant difference between clinical and ultrasonographic diagnosis. $(p<0.05)$.
Table (7): Comparison between clinical diagnosis before ultrasound and diagnosis after ultrasound of the studied patients.

\begin{tabular}{lcc}
\hline $\begin{array}{l}\text { Studied } \\
\text { Patients }\end{array}$ & $\begin{array}{l}\text { Clinical Diagnosis } \\
\text { (Before Ultrasound) }\end{array}$ & $\begin{array}{l}\text { Ultrasonography } \\
\text { (After Ultrasound) }\end{array}$ \\
\hline $\begin{array}{l}\text { Diagnosed Cases } \\
\text { n. }(\%)\end{array}$ & $16(53.33 \%)$ & $28(93.33 \%)$ \\
$\begin{array}{l}\text { Undiagnosed } \\
\text { Cases } \\
\text { n. }(\%)\end{array}$ & $14(46.67 \%)$ & $2(6.67 \%)$ \\
$p$-Value & \multicolumn{2}{c}{0.0005} \\
\hline
\end{tabular}

\section{Discussion}

Gynecological emergencies are disease conditions that threaten women life. Classically, the patient comes with acute pelvic and/or abdominal pain, with or without vaginal bleeding or discharge. Acute pelvic pain related to the gynecological tract is a common presentation in the emergency department.

The diagnosis was suspected on the basis of symptoms, and made by means of physical evaluation, including abdominal, pelvic, and bimanual gynecological examinations, laboratory tests, and transvaginal ultrasonography (TVS) and/or transabdominal ultrasonography (TAS).

Ultrasonography is the mainstay of imaging evaluation of patients presenting with gynecological emergencies (including acute pelvic pain and/or vaginal bleeding).

Advantages of the ultrasonography include absence of radiation, rapid availability, and excellent visualization of the pelvic organs. Improvements made in image quality have facilitated the rapid and accurate diagnosis of the majority of gynecological causes of acute pelvic pain.

Our study was carried out on 30 female patients presented with acute pelvic pain and/or vaginal bleeding. This study aimed to compare between the clinical provisional diagnosis and ultrasonography diagnosis of gynecological cases presenting in the Emergency Department of Tanta University Hospital.

\section{Complaint:}

In our study, about $76.67 \%$ of patients presented mainly with acute pelvic pain. About $23.33 \%$ of patients presented mainly with vaginal bleeding. In ectopic pregnancy, about $64.29 \%$ of patients presented with acute pelvic pain, and about $35.71 \%$ of patients presented with vaginal bleeding. 
On the same line, Tuli et al. [8] study found that abdominal pain, and vaginal bleeding were the usual presenting complaint of ectopic pregnancy patients in $92.82 \%$, and $73.80 \%$ of patients respectively.

Prasanna et al. [9] study which was in line with our study found that about $90 \%$ of ectopic pregnancy patients presented with abdominal pain, and about $68 \%$ of patients came with a history of bleeding per vagina.

\section{Final diagnosis:}

This study revealed the most common gynecological emergency was Ectopic Pregnancy (about $46.67 \%$ ). About $16.67 \%$ of included patients were diagnosed as Adnexal Torsion, about $13.33 \%$ of patients were diagnosed as Ovarian Cyst Rupture, about $13.33 \%$ of patients were diagnosed as PID. Uterine Mass represented only about $10 \%$ of patients in our study.

In Varas et al. [10] study the data of 365 patients with acute pelvic pain were analyzed, of whom 103 were confirmed to have gynecological emergencies. The most common emergencies were ectopic pregnancy, then ovarian cyst torsion, and came next complicated PID which is in line with our study.

Ectopic pregnancy was the 2 nd common diagnosis $(22 \%)$ of non-traumatic acute abdominal pain after acute appendicitis (out of our scope) in Ashaolu BA et al. [11] study.

A study by Toret-Labeeuw et al. [12] that included 234 female patients presented with acute pelvic pain, found that about $58 \%$ (136) of them was diagnosed as ectopic pregnancy. PID represented about $13 \%$ (31) of patients presented with acute pelvic pain. These results were in line with our finding.

\section{Diagnosis before and after ultrasonography:}

In all the studied patients, about $53.33 \%$ of patients were diagnosed by clinical assessment. After ultrasound, about $93.33 \%$ of patients were diagnosed with a statistically significant difference between both. $(p<0.05)$.

A study by Toret-Labeeuw et al. [12] showed that sensitivity of the clinical assessment (87\%) was lower than ultrasonography-aided diagnosis (99\%) for assessment of patients presented with acute pelvic pain but with a slight difference between both. This may be explained by the wide duration of the study (about 3 years), and a large population were included (about 234 patients).

Conclusion: Adding Ultrasonography as a bedside test was found to be superior to physical examination in diagnosis of acute pelvic pain. If a gynecologic disorder couldn't be confirmed, or the sonographic finding are equivocal, MultiDetector Computed Tomography (MDCT) is another imaging choice.

Ultrasound is generally accepted as the first imaging modality used in patients with acute pelvic pain. The true value of ultrasound in acute pelvic pain lies in its ability to detect gynecologic disorders.

Recommendation: Ultrasonography is an important tool for assessing and diagnosing gynecological emergencies. It should be used as a bedside test for patients presenting to the Emergency Department with acute pelvic pain and/or vaginal bleeding.

More studies are needed to compare the role of Transabdominal US (TAS) and Transvaginal US (TVS) in diagnosing gynecological emergencies.

Also, more studies are needed to compare role and sensitivity of ultrasonography, computed tomography (CT), magnetic resonance imaging (MRI) in patients presenting with acute pelvic pain for detection of gynecological emergencies.

\section{References}

1- RAMPHAL S.R. and MOODLEY J.: Emergency Gynaecology. Best Practice \& Research Clinical Obstetrics \& Gynaecology, 20 (5): 729-50, 2006.

2- JIVRAJ S. and FARKAS A.: Gynaecological Causes of Abdominal Pain. Surgery (Oxford), 33 (5): 226-30, 2015.

3- BOYD C.A. and RIALL T.S.: Unexpected Gynecological Findings during Abdominal Surgery. Current Problems in Surgery, 49 (4): 195-251, 2012.

4- GUPTA S. and MANYONDA I.T.: Acute Complications of Fibroids. Best Practice \& Research Clinical Obstetrics \& Gynaecology, 23 (5): 609-17, 2009.

5- MISE P.J., MISE S.J., MISE A., et al.: Role of Transvaginal Sonography in Various Gynecological Disorders. International Journal of Reproduction, Contraception, Obstetrics and Gynecology, 6 (7): 3138-42, 2017.

6- LAMBERT M.J. and VILLA M.: Gynecologic Ultrasound in Emergency Medicine. Emergency Medicine Clinics of North America, 22 (3): 683-96, 2004.

7- MAZZEI M.A., GUERRINI S., SQUITIERI N.C., et al.: The Role of US Examination in the Management of Acute Abdomen. Critical Ultrasound Journal, 1 (5): 19, 2013. 
8- TULI A.G., GOYAL S., LIVINGSTON D., et al.: Ectopic Pregnancy: A Five Year Retrospective Study in a Tertiary Care Hospital. International Journal of Reproduction, Contraception, Obstetrics and Gynecology, 4 (5): 14003, 2017.

9- PRASANNA B., JHANSI C.B., SWATHI K., et al.: A study on risk factors and Clinical Presentation of Ectopic Pregnancy in Women Attending a Tertiary Care Centre. International Association of Infant Massage, 3 (1): 90-6, 2016.

10- VARAS C., RAVIT M., MIMOUN C., et al.: Optimal Combination of Non-Invasive Tools for the Early Detection of Potentially Life-Threatening Emergencies in Gynecology. PLOS One, 11 (9): 1-12, 2016.

11- ASHAOLU B.A., ASALEYE M.C., ADETILOYE A.V., et al.: Spectrum of Diseases and Diagnostic Values of Ultrasound in Adult Patients with Nontraumatic Acute Abdomen in a Nigerian Tertiary Health Facility. Nigerian Journal of Surgery, 21 (1): 6-12, 2015.

12- TORET-LABEEUW F., HUCHON C., POPOWSKI T., et al.: Routine Ultrasound Examination by Ob/Gyn Residents Increase the Accuracy of Diagnosis for Emergency Surgery in Gynecology. World Journal of Emergency Surgery, 8 (1): 16-23, 2013.

\title{
مقارنة بين التشخيص الإكلينيكى والتصوير بالموجات فوق الصوتية فى حالات الطوارىى في والئى علم النساء
}

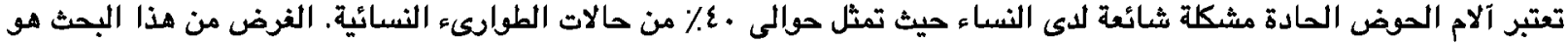

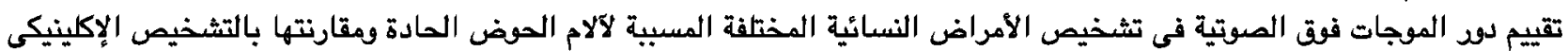 \\ لمالات من خلال الفصص الإكلينيكى والتحاليل المعملية.

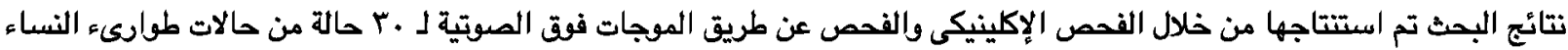

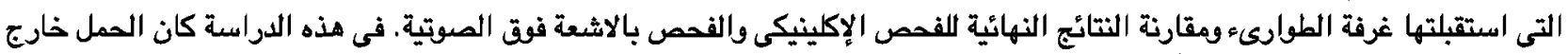

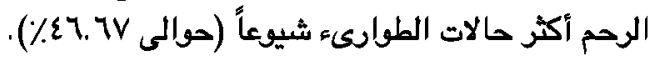

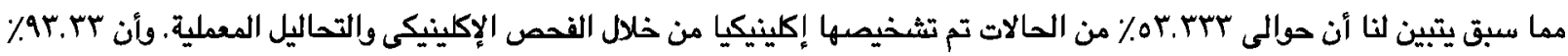 \\ تم تثخيصها من خلال الفحص بالموجات فوق الصوتية. من الصاتية \\ مما سبق يتبين أهمية الموجات فوق الصوتية فى الوصول للتشخيص النهائى فى حالات طوارىء النساء.
}

